MGM promot er met hyl at i on and tenozol om de response in chor oi d pl exus car ci noma

\begin{tabular}{|l|l|}
\hline 著者 & $\begin{array}{l}\text { M saki Koui chi, Nakada M t sut oshi, Mohr i } \\
\text { Nasanao, Hay ashi Yut aka, Hanada Jun- } \mathrm{i} \text { chi r o }\end{array}$ \\
\hline $\begin{array}{l}\text { j our nal or } \\
\text { publ i cat i on t i t l e }\end{array}$ & Br ai n Tumor Pat hol ogy \\
\hline vol une & 28 \\
\hline number & 3 \\
\hline page r ange & $259-263$ \\
\hline year & $2011-07-01$ \\
\hline URL & ht t p: //hdl . handl e. net /2297/29468 \\
\hline
\end{tabular}




\title{
MGMT promoter methylation and temozolomide response in choroid plexus carcinoma
}

\author{
Kouichi Misaki $\cdot$ Mitsutoshi Nakada \\ Masanao Mohri • Yutaka Hayashi • \\ Jun-ichiro Hamada
}

Received: 30 October 2010/Accepted: 8 March 2011/Published online: 26 March 2011

(C) The Japan Society of Brain Tumor Pathology 2011

\begin{abstract}
Choroid plexus carcinoma (CPC) is a malignant tumor with a strong tendency to spread along the cerebrospinal fluid pathway. There is no standardized chemotherapy protocol for this rare tumor. We report a 38-year-old man with CPC in the lateral ventricle with obstructive hydrocephalus. Because of the poor demarcation between thalamus and fornix, subtotal tumor resection was performed. Postoperative spine magnetic resonance (MR) image revealed whole spinal axis dissemination. After diagnosis of CPC, the patient was treated with whole ventricular and spine radiation concomitant with temozolomide chemotherapy, although the $O_{6}$-methylguanineDNA methyltransferase (MGMT) promoter was found to be unmethylated. Although MR images revealed transient stable disease during adjuvant therapy, tumor progression was depicted after four cycles of temozolomide therapy. We discuss the ineffectiveness of adjuvant temozolomide therapy for CPC in connection with $\mathrm{O}_{6}$-methylguanineDNA methyltransferase promoter methylation.
\end{abstract}

Keywords Choroid plexus carcinoma - Temozolomide . MGMT $\cdot$ Methylation

\section{Introduction}

Choroid plexus tumors (CPT) are rare intraventricular papillary neoplasms, comprising $0.4-1.0 \%$ of primary

K. Misaki $(\varangle) \cdot$ M. Nakada · M. Mohri · Y. Hayashi .

J. Hamada

Department of Neurosurgery, Division of Neuroscience, Graduate School of Medical Science, Kanazawa University,

13-1 Takara-machi, Kanazawa, Ishikawa 920-8641, Japan

e-mail: misaki@ns.m.kanazawa-u.ac.jp brain neoplasms and predominantly occurring in young children [1-5]. Choroid plexus carcinoma (CPC), which shows histological malignancy, represents between $8 \%$ and $25 \%$ of all CPTs $[2,3,6-8]$. The outcome of benign choroid plexus papilloma (CPP) is usually excellent following gross total resection, whereas the more aggressive biological behavior of CPC necessitates postoperative adjuvant treatment $[1,9]$. Chemotherapy has led to remission of choroid plexus carcinoma but does not always improve survival or avert recurrence [1, 10-12]. The DNA-alkylating agent temozolomide is effective for malignant gliomas, whereas evidence for its efficacy for CPT remains unclear [7, 12-14]. Promoter methylation of the $O_{6}$-methylguanine-DNA methyltransferase (MGMT) DNA-repair gene is a marker for the response to alkylating agents in malignant gliomas, although this has not been probed in CPT [13-15]. Herein, we report an adult case of CPC with craniospinal seeding who underwent subtotal resection followed by administration of temozolomide with assessment of MGMT promoter methylation status.

\section{Case report}

\section{Clinical course}

A 38-year-old previously healthy man presented with a 5-month history of intermittent headache and memory impairment. Magnetic resonance (MR) images demonstrated a heterogeneously enhanced mass that showed bilateral extension from the septum pellucidum with obstructive hydrocephalus (Fig. 1a, b). He underwent tumor resection via the interhemispheric transcallosal approach. A small part of the mass was left because it was adhered to the fornix and thalamus (Fig. 1c). Hydrocephalus was treated 
with a ventriculoperitoneal shunt. Radiation therapy was initiated 3 weeks after tumor resection with administration of temozolomide as described later. Three months after tumor removal, spine MR images demonstrated entire spinal axis dissemination with the largest lesion at the level of T3; at that time, the patient suffered from progressive paraparesis (Fig. 1d, e). Chest, abdominal, and pelvic computed tomography excluded systemic malignancy.

\section{Pathological findings}

Pathologically, the tumor showed blurring of the papillary pattern, which was composed of atypical epithelial cells with brain invasion (Fig. 2a, b). There was brisk mitotic activity with an MIB-1 staining index of $14.2 \%$ (Fig. 2c). Tumor cells were positive for cytokeratin, epithelial membrane antigen (EMA), and carcinoembryonic antigen (CEA) (Fig. 2d), but negative for transthyretin, S-100 protein, glial fibrillary acidic protein (GFAP), and synaptophysin. The tumor was diagnosed as a CPC.

\section{Analysis of MGMT promoter methylation}

The methylation-specific polymerase chain reaction (PCR) assay, known as MSP, was performed to evaluate the methylation status of MGMT promoter in the tumor tissue. DNA was extracted from the formalin-fixed, paraffinembedded tissue sample. After bisulfate modification of the DNA, the MSP assay was performed at least three times. In the case of our patient, the MGMT promoter was found to be unmethylated (Fig. 3) [13, 14, 16, 17].

Adjuvant therapy

Combined radiation and chemotherapy were performed. The tumor bed, whole ventricular, and whole spine were treated with 60, 30, and $30 \mathrm{~Gy}$, respectively. The patient also received simultaneous adjuvant temozolomide therapy at $75 \mathrm{mg} / \mathrm{m}^{2} /$ day (140 mg daily). Four weeks after completion of radiation therapy, the temozolomide dose was increased to $150 \mathrm{mg} / \mathrm{m}^{2} /$ day (260 mg daily) for 5 consecutive days per 4 weeks; four cycles were completed without significant toxicity. Although MR images during the adjuvant therapy revealed transient stable disease, multifocal spread of tumor was depicted at the final chemotherapy. The patient died of respiratory failure caused by dissemination around the brainstem 11 months after the initial surgery (see Fig. 1f).

\section{Discussion}

Histologically, CPP corresponds to World Health Organization (WHO) grade I, atypical CPP, which is defined as CPP with increased mitotic activity to grade II and CPC to WHO grade III. CPC shows frank signs of malignancy, including nuclear pleomorphism, frequent mitoses, high nucleus-to-cytoplasm ratios, increased cellular density,
Fig. 1 Brain and spine $\mathrm{T}_{1}$-weighted magnetic resonance (MR) images with gadolinium contrast. a, b Axial (a) and coronal (b) images demonstrated the heterogeneous mass extending bilaterally from the septum pellucidum. The tumor was directly contiguous with the bilateral thalami. c Postoperative coronal image showed a small residual tumor between the bilateral thalami (arrow). d, e Sagittal spine MR images revealed multiple subarachnoid enhanced lesions in the whole spinal axis (arrows). f Sagittal image of craniocervical junction demonstrated multiple seeding lesions around brainstem and cervical cord (arrows)
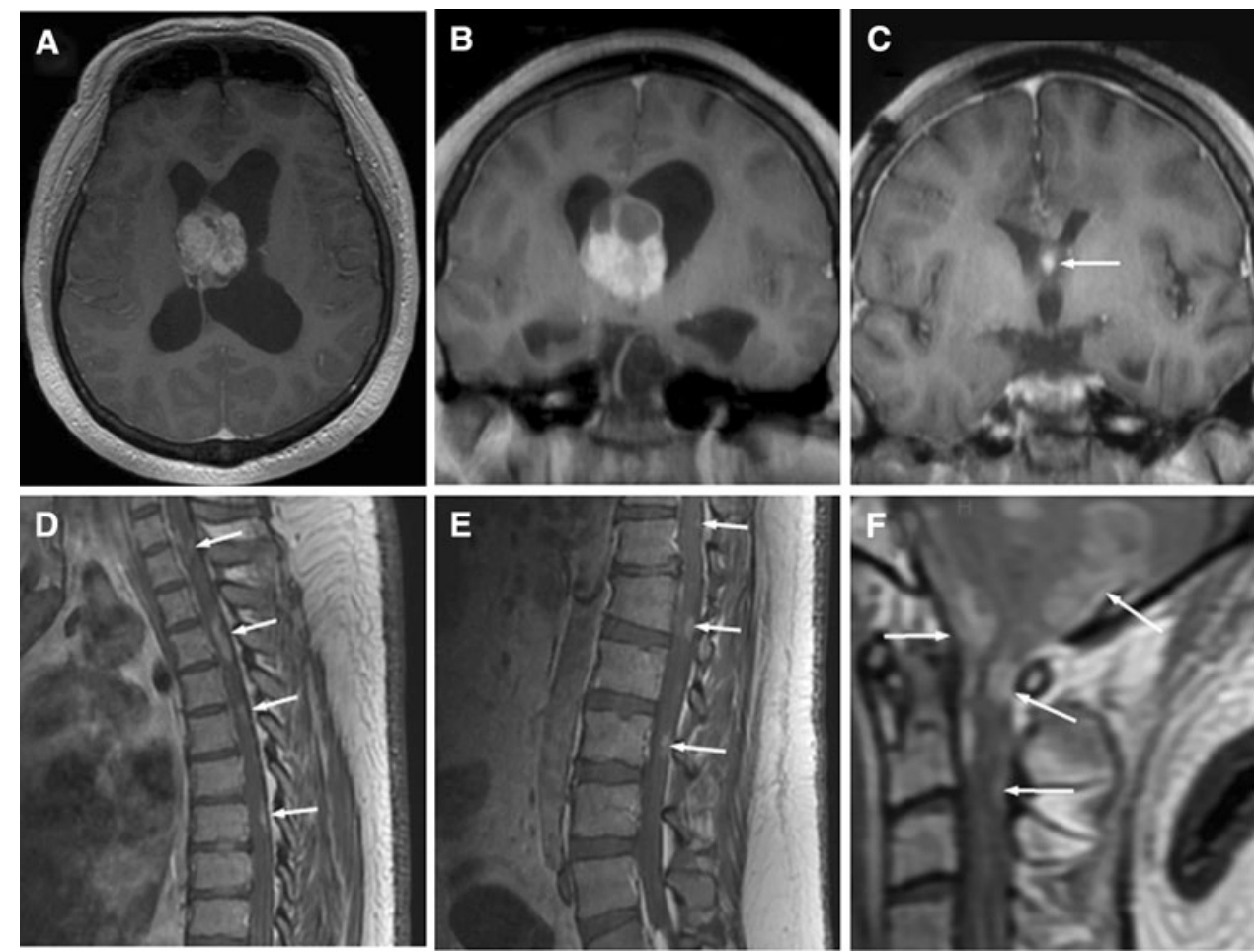
Fig. 2 a Hematoxylin and eosin staining showed atypical epithelial cells arranged in papillary-like projections, with abundant mitoses. b Tumor showed invasion to adjacent brain. c MIB-1 staining index was $14.2 \%$. d Partial cytokeratin positivity was demonstrated in the tumor cells. Bars $100 \mu \mathrm{m}$
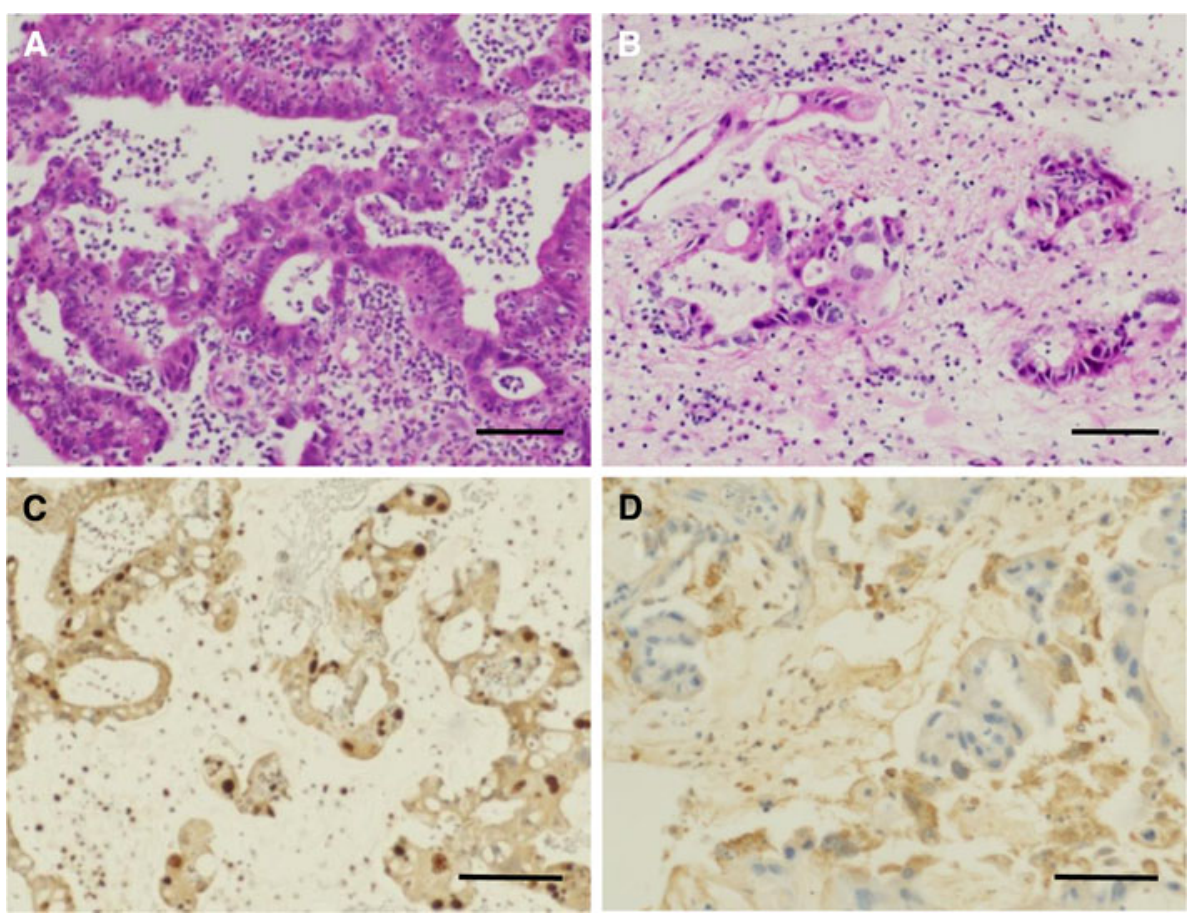

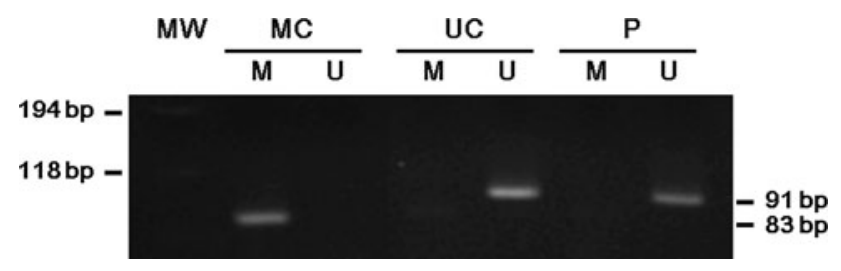

Fig. 3 Polymerase chain reaction (PCR) products in the $M$ lanes indicate methylation of $\mathrm{O}_{6}$-methylguanine-DNA methyltransferase $(M G M T)$ promoter. $M C$, U87 cell line as methylated control; $U C$, U138MG cell line as unmethylated control; $P$, patient's tumor sample; $M W$, molecular weight marker

blurring of the papillary pattern with poorly structured sheets of tumor cells, necrotic areas, and often diffuse brain invasion [5]. The reported mean MIB-1 staining index is $1.9 \%$ for CPP and $13.8 \%$ for CPC [5]. Immunohistochemically, cytokeratin and vimentin are frequently expressed in CPP and CPC whereas transthyretin, which is a specific marker for CPT, S-100 protein, and GFAP, is less frequently expressed in CPC than in CPP [5, 18, 19]. Immunodetection of CEA and EMA is variously reported in CPT $[5,19,20]$. The differential diagnosis of CPC includes metastatic adenocarcinomas and anaplastic ependymomas $[4,21,22]$. The present case did not have primary systemic tumor and perivascular pseudorosettes and showed evidence of frequent mitoses, brain invasion, and expression of cytokeratin; these findings were consistent with CPC. Negative staining for transthyretin and S-100 protein such as the present case is reported to correlate with poor prognosis in CPT [19].
In the treatment of CPC, the extent of surgery is a significant prognostic factor $[1,6,8,10,11,23]$. Packer et al. [11] reported relapse in five of six patients who had partial resection for CPC compared to one of five patients with total resection. A meta-analysis revealed that CPC patients had a 2-year survival rate of $72 \%$ and $34 \%$ for those with gross total resection and those with incomplete resection, respectively [1]. Although aggressive surgical resection of the tumor is essential for long survival, radical resection is restricted by the tendency to invade adjacent brain [18] The present case could not achieve gross total resection because of tumor adhesion to fornix and thalamus. In cases of incomplete tumor removal or craniospinal metastasis, postoperative adjuvant therapy is strongly recommended.

CPT has a tendency to cause metastases along cerebrospinal fluid pathways [4, 18, 23-28]. A review of CPT showed that the incidence of metastasis is $12-50 \%$ in its clinical course $[1,23]$. Metastasis is more frequent in CPC and a significant factor affecting the survival time in CPT $[1,23]$. Dissemination is also detected postoperatively, as in the present case $[7,18,27,28]$. Because spine MR imaging was not performed before the initial surgery, it remains unclear whether surgery was responsible for tumor cell seeding, which is described as drop metastasis.

Several reports demonstrated that postoperative irradiation confers a survival advantage in patients who have undergone a subtotal resection as well as gross total resection $[1,9,10,24,29]$. Some authors recommend regional radiation [30] whereas others performed craniospinal radiation [11, 24]. Information concerning 
Table 1 Summary of temozolomide therapy for choroid plexus tumors

\begin{tabular}{llllllll}
\hline References & Age/sex & Pathology & Site of origin & Dissemination & MGMT promoter & $\begin{array}{l}\text { Temozolomide } \\
\begin{array}{l}\text { Follow-up } \\
\text { (after dissemination) }\end{array}\end{array}$ \\
\hline McCall [7] & $30 / \mathrm{F}$ & CPP & Fourth ventricle & Yes & NA & 12 cycles & 17 months: stable \\
& 22/F & CPP & Fourth ventricle & Yes & NA & 18 cycles & 4 years: progression \\
Lozier [12] & 68/F & CPC & Temporal lobe & No & NA & 6 cycles & 44 months: stable \\
Present case & 38/M & CPC & Lateral ventricle & Yes & Unmethylated & 4 cycles & 11 months: dead \\
\hline
\end{tabular}

$C P C$ choroid plexus carcinoma, $C P P$ choroid plexus papilloma, $M G M T O_{6}$-methylguanine-DNA methyltransferase, $N A$ not available

appropriate doses and extent of radiation needed is still lacking $[12,18]$. For CPC patients, because dissemination and relapses outside the primary site are likely to occur, craniospinal radiation therapy should be considered, especially in adults who have less risk of intellectual sequelae caused by radiation than do children [11]. In the present case, the patient showed preoperative memory impairment caused by tumor invasion to the fornix. Radiation-induced encephalopathy causes cognitive disturbances such as mental slowing and deficits in attention and memory in adults as well as children [31]. Kleinberg et al. [32] demonstrated that adult patients receiving partial brain irradiation tended to retain better memory functions than those receiving whole brain treatment. Therefore, we selected whole ventricular radiation instead of whole brain radiation to avoid progression of intellectual disorder. Although the overall survival of CPC patients resected partially with craniospinal irradiation was reported to be significantly better than that without irradiation, the 2-year overall survival rate of those with irradiation was only about $50 \%$ [1]. This result indicated requirement of adjuvant chemotherapy, particularly for CPC patients with obvious dissemination such as the present case.

There is no standardized chemotherapy protocol for this rare tumor [1, 10-12]. Although meta-analysis of CPC demonstrated that etoposide was the most effective drug, the response rate was only less than half of cases [33]. Several reports demonstrated efficacy of temozolomide for CPTs (Table 1) [7, 12]. In two cases of recurrent CPP with craniospinal dissemination treated with temozolomide, one case showed radiographically stable disease for 17 months after surgery, whereas in the other case tumor control failed [7]. In one case of adult CPC without dissemination treated with temozolomide after gross total resection, no evidence of recurrence was seen for 44 months after surgery [12]. However, these cases received temozolomide chemotherapy without analysis of MGMT promoter methylation. Hasselblatt et al. [15] reported that aberrant methylation of MGMT promoter was confirmed in seven of eight cases in CPC without evaluation of the temozolomide efficacy. Our case may be rare with lack of methylation of the MGMT promoter. As there were no other potent chemotherapeutic agents and no evidence of MGMT promoter methylation status as to temozolomide efficacy in CPC, we administered temozolomide for the present case. Unfortunately, our case did not receive any benefit from temozolomide, which may suggest that unmethylation of the MGMT promoter predicts the ineffectiveness of temozolomide for CPC as well as glioblastoma. The ineffectiveness of temozolomide might also be lack of a DNA mismatch-repair system that recognizes DNA mismatches caused by temozolomide and repairs resynthesis of DNA. The DNA mismatch-repair system causes repeated futile mismatch-repair cycles, creating DNA strand breaks and cytotoxicity. The lack of this system leads to ineffectiveness of temozolomide [34]. To our best knowledge, this is the first report of a CPC that was treated with temozolomide, coupled with assessment of MGMT promoter methylation. A large population of CPC cases will be needed to confirm this speculation.

Acknowledgments This report is not associated with any financial support or grants.

\section{References}

1. Wolff JE, Sajedi M, Brant R, Coppes MJ, Egeler RM (2002) Choroid plexus tumours. Br J Cancer 87:1086-1091

2. Pascual-Castroviejo I, Villarejo F, Perez-Higueras A, Morales C, Pascual-Pascual SI (1983) Childhood choroid plexus neoplasms. A study of 14 cases less than 2 years old. Eur J Pediatr 140:51-56

3. Matsuda M, Uzura S, Nakasu S, Handa J (1991) Primary carcinoma of the choroid plexus in the lateral ventricle. Surg Neurol 36:294-299

4. Dohrmann GJ, Collias JC (1975) Choroid plexus carcinoma. J Neurosurg 43:225-232

5. Rickert CH, Paulus W (2001) Tumors of the choroid plexus. Microsc Res Tech 52:104-111

6. Connor SE, Chandler C, Bodi I, Robinson S, Jarosz JM (2002) Preoperative and early postoperative magnetic resonance imaging in two cases of childhood choroid plexus carcinoma. Eur Radiol 12:883-888

7. McCall T, Binning M, Blumenthal DT, Jensen RL (2006) Variations of disseminated choroid plexus papilloma: 2 case reports and a review of the literature. Surg Neurol 66:62-67; discussion 67-68

8. Fitzpatrick LK, Aronson LJ, Cohen KJ (2002) Is there a requirement for adjuvant therapy for choroid plexus carcinoma that has been completely resected? J Neuro-Oncol 57:123-126 
9. Wolff JE, Sajedi M, Coppes MJ, Anderson RA, Egeler RM (1999) Radiation therapy and survival in choroid plexus carcinoma. Lancet 353:2126

10. Wyatt SS, Price RA, Holthouse D, Elsaleh H (2001) Choroid plexus carcinoma in an adult. Australas Radiol 45:369-371

11. Packer RJ, Perilongo G, Johnson D, Sutton LN, Vezina G, Zimmerman RA, Ryan J, Reaman G, Schut L (1992) Choroid plexus carcinoma of childhood. Cancer (Phila) 69:580-585

12. Lozier AP, Arbaje YM, Scheithauer BW (2009) Supratentorial, extraventricular choroid plexus carcinoma in an adult: case report. Neurosurgery 65:E816-E817

13. Hegi ME, Diserens AC, Godard S, Dietrich PY, Regli L, Ostermann S, Otten P, Van Melle G, de Tribolet N, Stupp R (2004) Clinical trial substantiates the predictive value of O-6-methylguanine-DNA methyltransferase promoter methylation in glioblastoma patients treated with temozolomide. Clin Cancer Res 10:1871-1874

14. Hegi ME, Diserens AC, Gorlia T, Hamou MF, de Tribolet N, Weller M, Kros JM, Hainfellner JA, Mason W, Mariani L, Bromberg JE, Hau P, Mirimanoff RO, Cairncross JG, Janzer RC, Stupp R (2005) MGMT gene silencing and benefit from temozolomide in glioblastoma. N Engl J Med 352:997-1003

15. Hasselblatt M, Muhlisch J, Wrede B, Kallinger B, Jeibmann A, Peters O, Kutluk T, Wolff JE, Paulus W, Fruhwald MC (2009) Aberrant MGMT (O6-methylguanine-DNA methyltransferase) promoter methylation in choroid plexus tumors. J Neuro-Oncol 91:151-155

16. Herman JG, Graff JR, Myohanen S, Nelkin BD, Baylin SB (1996) Methylation-specific PCR: a novel PCR assay for methylation status of $\mathrm{CpG}$ islands. Proc Natl Acad Sci USA 93:9821-9826

17. Palmisano WA, Divine KK, Saccomanno G, Gilliland FD, Baylin SB, Herman JG, Belinsky SA (2000) Predicting lung cancer by detecting aberrant promoter methylation in sputum. Cancer Res 60:5954-5958

18. Kang HS, Wang KC, Kim YM, Kim IO, Kim SK, Chi JG, Cho BK (1997) Choroid plexus carcinoma in an infant. J Korean Med Sci 12:162-167

19. Paulus W, Janisch W (1990) Clinicopathologic correlations in epithelial choroid plexus neoplasms: a study of 52 cases. Acta Neuropathol (Berl) 80:635-641

20. Barreto AS, Vassallo J, Queiroz Lde S (2004) Papillomas and carcinomas of the choroid plexus: histological and immunohistochemical studies and comparison with normal fetal choroid plexus. Arq Neuropsiquiatr 62:600-607
21. Valladares JB, Perry RH, Kalbag RM (1980) Malignant choroid plexus papilloma with extraneural metastasis. Case report. J Neurosurg 52:251-255

22. Singh A, Vermani S, Shruti S (2009) Choroid plexus carcinoma: report of two cases. Indian J Pathol Microbiol 52:405-407

23. Berger C, Thiesse P, Lellouch-Tubiana A, Kalifa C, Pierre-Kahn A, Bouffet E (1998) Choroid plexus carcinomas in childhood: clinical features and prognostic factors. Neurosurgery 42:470-475

24. Ausman JI, Shrontz C, Chason J, Knighton RS, Pak H, Patel S (1984) Aggressive choroid plexus papilloma. Surg Neurol 22:472-476

25. Cila A, Ozturk C, Senaati S (1992) Bilateral choroid plexus carcinoma of the lateral ventricles. US, CT and MR findings. Pediatr Radiol 22:136-137

26. Donovan DJ, Prauner RD (2005) Shunt-related abdominal metastases in a child with choroid plexus carcinoma: case report. Neurosurgery 56:E412; discussion E412

27. Imaya H, Kudo M (1991) Malignant choroid plexus papilloma of the IV ventricle. Childs Nerv Syst 7:109-111

28. Vaquero J, Cabezudo J, Leunda G, Carrillo R, Garcia Uria J (1979) Primary carcinoma of the choroid plexus with metastatic dissemination within the central nervous system. Acta Neurochir (Wien) 51:105-111

29. Wrede B, Liu P, Wolff JE (2007) Chemotherapy improves the survival of patients with choroid plexus carcinoma: a metaanalysis of individual cases with choroid plexus tumors. J NeuroOncol 85:345-351

30. Allen J, Wisoff J, Helson L, Pearce J, Arenson E (1992) Choroid plexus carcinoma: responses to chemotherapy alone in newly diagnosed young children. J Neuro-Oncol 12:69-74

31. Taphoorn MJ, Klein M (2004) Cognitive deficits in adult patients with brain tumours. Lancet Neurol 3:159-168

32. Kleinberg L, Wallner K, Malkin MG (1993) Good performance status of long-term disease-free survivors of intracranial gliomas. Int J Radiat Oncol Biol Phys 26:129-133

33. Berrak SG, Liu DD, Wrede B, Wolff JE (2010) Which therapy works better in choroid plexus carcinomas? J Neuro-Oncol (Epub ahead of print)

34. Hirose Y, Katayama M, Stokoe D, Haas-Kogan DA, Berger MS, Pieper RO (2003) The p38 mitogen-activated protein kinase pathway links the DNA mismatch repair system to the $G_{2}$ checkpoint and to resistance to chemotherapeutic DNA-methylating agents. Mol Cell Biol 23:8306-8315 